\title{
Electric Dipole Transition Parameters for Low-Lying Levels for Neutral Actinium
}

\author{
L. ÖZDEMiR* AND G. ÜRER \\ Physics Department, Sakarya University, 54187, Sakarya, Turkey \\ (Received November 19, 2009; revised version April 20, 2010; in final form May 4, 2010)
}

\begin{abstract}
We have calculated the energies of some low-lying levels $\left(6 d 7 s^{2}, 6 d^{2} 7 s, 6 d 7 s 7 p\right.$ and $\left.6 d^{2} 7 p\right)$ and electric dipole transition parameters (wavelengths, oscillator strengths and transition probabilities) between them for neutral actinium $(Z=89)$. In the calculations, the correlation and relativistic effects are considered by multiconfiguration Hartree-Fock method within the framework Breit-Pauli Hamiltonian. The results obtained are compared with the available experimental and theoretical works in the literature.
\end{abstract}

PACS numbers: 31.15.ag, 31.15.aj, 31.30.-i, 32.70.Cs

\section{Introduction}

Some atomic properties as energies (ionization potentials and excitation energies), wavelengths, oscillator strengths, and transition probabilities are theoretically and experimentally known for most atoms. This information is still very fragmentary, especially for rareearth atoms including lanthanides $(Z=57$ to 70$)$ and actinides $(Z=89$ to 102$)$ having the $4 f$ and $5 f$ subshells. These series have been frequently called as rare-earth atoms. The spectra of rare-earth atoms, especially actinides series, are tremendously complex, and one spectrum may contain tens or hundreds of thousands of observable lines. The difficulty in observing actinide elements arises from configuration interactions in their spectra. In many cases, a large amount of data is required for a correct interpretation of the spectra, and, hence, a large amount of sample material is needed. Another complexity is capacity of computers. Large scale atomic structure computations require the repeated evaluation of many interaction matrix elements, each of which is the product of a radial and an angular integral. The results of the angular integrations do not change as the radial functions are adjusted during the course of a calculation, so they are typically computed at the beginning and stored. However, quantity of this angular data grows rapidly with the size of the computer programs capable of producing accurate atomic data, the storage and retrieval of angular data is becoming a serious issue.

The actinide series have valence subshells running from $5 f^{1}$ to $5 f^{14}$. Neutral actinium (Ac I ) occupies a particular place in periodic table and opens the actinide series although it is not always regarded as belonging to the actinides because of the lack of a $5 f$ electron in

\footnotetext{
* corresponding author; e-mail: lozdemir@sakarya.edu.tr
}

the ground configuration. The $5 f$ orbitals tend to be more diffuse and to lie deeply buried in the core of the corresponding $4 f$ orbital in the lanthanides. The spectra of actinides are more complicated than of the lanthanides. The information on transition energies and radiative transitions is more fragmentary and scarce for actinides due to these complex spectra. Some lines for Ac I were classified on internet web site [1] and [2]. The configuration interaction parameters were calculated for some heavy atoms by Judd [3]. The theoretical treatment of energy differences between lowest energy levels for some lanthanide and actinide vapors were investigated by Nugent et al. and Vander Sluis et al. $[4,5]$. Brewer reported energies of the electronic configurations of the gaseous ions of the lanthanides and actinides [6]. Desclaux calculated the relativistic expectation values for $Z=1$ to $Z=120$ by relativistic Dirac-Fock method [7]. The Hartree-Fock calculations of the electronic structure and energies from hydrogen to lawrencium with two open shells were made by Mann [8]. The binding energies for actinides were given using Hartree-Fock (HFR) method by Rajnak [9]. Köhler et al. reported the first ionization potentials of some actinides elements by resonance ionization mass spectroscopy [10]. Transition energies of lanthanum, actinium, and eka-actinium were obtained by the relativistic coupled cluster method by Eliav et al. [11]. The calculation of $f-f$ spectra of lanthanide and actinide ions by the multiconfiguration Dirac-Fock configuration interaction method was performed by Seth et al. [12]. Petrov et al. analyzed the Breit interaction in relativistic effective core potential calculations of actinides [13]. Resonance line oscillator strengths for atomic absorption analysis were given by Doidge [14]. Ionization energies of the neutral actinides were reported by Sugar [15] (the values belonging to the revised version of this study can be found in [16] and [2]). 
TABLE I

Configurations for even- and odd-parity levels for electric dipole transitions in Ac I .

\begin{tabular}{|c|c|c|}
\hline \multicolumn{3}{|c|}{ Configurations } \\
\hline$A$ & $B$ & $C$ \\
\hline \multicolumn{3}{|c|}{ For even-parity: } \\
\hline $\begin{array}{l}6 d n s^{2}, 6 d^{2} n s, 6 d n p^{2}, 7 s n p^{2}, n p^{2} 8 s \\
(n=7,8), \quad 7 s^{2} 8 s, \quad 7 s 8 s^{2}, \quad 6 d^{3}, \\
6 d 7 s 8 s, 6 d 7 p 8 p, 7 s 7 p 8 p, 7 p 8 s 8 p\end{array}$ & $\begin{array}{l}\text { All of the even-parity configura- } \\
\text { tions in } A+7 s 5 f n p(n=7,8) \text {, } \\
6 d 5 f^{2}, 5 f 7 p 8 s, 5 f 8 s 8 p\end{array}$ & $\begin{array}{l}\text { All of the even-parity configura- } \\
\text { tions in } B+7 s^{2} 5 g, 6 d 7 s 5 g, 6 d^{2} 5 g\end{array}$ \\
\hline \multicolumn{3}{|c|}{ For odd-parity: } \\
\hline $\begin{array}{l}6 d 7 s n p, 6 d^{2} n p, 7 s^{2} n p, n p^{3}, n p 8 s^{2} \\
(n=7,8), 7 s 7 p 8 s, 6 d 7 p 8 s, 6 d 8 s 8 p \\
7 s 8 s 8 p, 7 p^{2} 8 p, 7 p 8 p^{2}\end{array}$ & $\begin{array}{l}\text { All of the odd-parity configura- } \\
\text { tions in } A+6 d n s 5 f(n=7,8) \text {, } \\
7 s^{2} 5 f, n d^{2} 5 f(n=6,7)\end{array}$ & $\begin{array}{l}\text { All of the odd-parity configura- } \\
\text { tions in } B+6 d 7 p 5 g, 7 s 7 p 5 g\end{array}$ \\
\hline
\end{tabular}

The goal of this paper was to find atomic data for neutral actinium $(Z=89)$ and apply the MCHF method [17] containing the correlation and relativistic effects (in the framework Breit-Pauli Hamiltonian) for this atom. We made similar atomic structure calculations for lanthanum which has ground state $5 d 6 s^{2}{ }^{2} D_{3 / 2}$ outside the core [Xe] in our previous works [18-20]. First, we have here calculated the first ionization potential for neutral actinium. The first ionization potential for an element is a fundamental physical and chemical property. The accurate determination of ionization potential aids in identifying systematic trends in binding energies and in the interpretation of atomic spectra. Actinium has the ground state $6 d 7 s^{2}{ }^{2} D_{3 / 2}$ outside the core [Rn]. As known, the Breit effects give a very significant contribution to the physical and chemical properties of heavy elements. To consider correlation effects, we have selected three various configuration sets outside the core [Rn] denoted by $A, B$, and $C$ in Table I. The calculations have been performed by the MCHF code [21]. Here, we have presented the energies of low-lying levels and the electric dipole transition parameters, including wavelengths, oscillator strengths and transition probabilities, between them according to $A$, $B$, and $C$ configuration sets. Transition parameters are fundamental quantities for many scientific applications. These data will be useful to predict and interpret the spectral output of Ac I .

\section{Method of calculation}

The atomic state is described by a wave function that is the solution of the wave equation,

$$
(H-E) \Psi=0,
$$

where $H$ is the Hamiltonian and $E$ is the total energy for the system. In the non-relativistic approximation, $H$ is described in the form (in atomic units),

$$
H_{\mathrm{NR}}=-\frac{1}{2} \sum_{i=1}^{N}\left(\nabla_{i}^{2}+\frac{2 Z}{r_{i}}\right)+\sum_{i<j} \frac{1}{r_{i j}}
$$

where $Z$ is the nuclear charge of atom; $r_{i}$ and $r_{i j}$ is the distance of the $i$-th electron from the nucleus, and between the electron $i$ and electron $j$, respectively. In the MCHF approximation [17] the wave function $\Psi$ is ex- panded as a linear combination of orthonormal configuration state functions (CSFs),

$$
\Psi(\gamma L S)=\sum_{i=1}^{M} c_{i} \Phi\left(\gamma_{i} L S\right), \quad \sum_{i=1}^{M} c_{i}^{2}=1,
$$

where $\Phi\left(\gamma_{i} L S\right), \gamma_{i}$ and $c_{i}$ denote configuration state function in $L S$ coupling, configurations and mixing (or expansion) coefficients, respectively. Each CSF is a vector coupled state of one-electron orbitals,

$$
\Phi_{n l m_{l} m_{s}}(r, \theta, \phi, \sigma)=\frac{1}{r} P_{n l}(r) Y_{l m_{l}}(\theta, \phi) \chi_{m_{s}}(\sigma),
$$

where the spherical harmonics $\left(Y_{l m_{l}}\right)$ and spinors $\chi_{m_{s}}$ are known. The radial functions $P_{n l}(r)$ may be known functions such as hydrogenic or may need to be determined. In the MCHF approximation, the radial functions are determined in the non-relativistic approximation. Thus, the total energy is given by

$$
E=\left\langle\psi(\gamma L S)\left|H_{\mathrm{NR}}\right| \psi(\gamma L S)\right\rangle=\sum_{i j} c_{i} c_{j} H_{i j},
$$

where $H_{\mathrm{NR}}$ is the Hamiltonian matrix (interaction matrix) with elements

$$
H_{i j}=\left\langle\Phi\left(\gamma_{i} L S\right)\left|H_{\mathrm{NR}}\right| \Phi\left(\gamma_{j} L S\right)\right\rangle .
$$

Thus, in the MCHF calculations, only coefficients are optimized.

In the Breit-Pauli approximation, the Hamiltonian is expanded to include relativistic corrections. The BreitPauli Hamiltonian is a first order perturbation correction, $(\alpha Z)^{2}$, to the non-relativistic Hamiltonian. This Hamiltonian is written as a sum in the form

$$
H_{\mathrm{BP}}=H_{\mathrm{NR}}+H_{\mathrm{R}},
$$

where $H_{\mathrm{R}}$ is the relativistic Hamiltonian. This Hamiltonian can be divided,

$$
H_{\mathrm{R}}=H_{\mathrm{RS}}+H_{\mathrm{FS}}
$$

where $H_{\mathrm{RS}}$ is the relativistic shift operator including mass correction $\left(H_{\mathrm{MC}}\right)$, the one- and two-body Darwin terms $\left(H_{\text {Darwin }}\right)$, spin-spin contact $\left(H_{\mathrm{SSC}}\right)$ and orbit-orbit terms $\left(H_{\mathrm{OO}}\right)$. These corrections shift non-relativistic energy without any splitting of the levels.

$H_{\mathrm{FS}}$ is the fine structure operator including nuclear spin-orbit $\left(H_{\mathrm{SO}}\right)$, spin-other-orbit $\left(H_{\mathrm{SOO}}\right)$ and spin-spin terms $\left(H_{\mathrm{SS}}\right)$. The fine structure contributions split the non-relativistic energy to closely-spared levels. 
In MCHF approximation, the total energy is an eigenvalue of the $H_{\mathrm{NR}}$, and the expansion coefficients of the wave function form the corresponding eigenvector,

$$
(\boldsymbol{H}-E) \boldsymbol{c}=0
$$

where

$$
\boldsymbol{H}=H_{i j} \text {. }
$$

In the Breit-Pauli Hamiltonian, $L$ and $S$ are coupled to form a total angular momentum $J$. Thus, the MCHF atomic structure package [21] assumes that the radial functions are known and the wave function is a sum of configuration states for possibly different $L S$ terms and has the form

$$
\Psi(\gamma J)=\sum_{L S} \sum_{j} c_{j}(L S) \Phi\left(\gamma_{j} L S J\right) .
$$

The CSFs, $\Phi\left(\gamma_{j} L S J\right)$, for a configuration and coupling $\gamma_{j}$, term $L S$, and total angular momenta $L$ and $S$ coupled to $J$, are built from a basis of one-electron spin-orbitals. The expansion coefficients, $c_{j}(L S)$ and corresponding energy $E(L S J)$, are an eigenvector and eigenvalue, respectively, of the interaction matrix of these CSFs as defined by the Breit-Pauli Hamiltonian.

The radiative transition parameters (line strengths, oscillator strengths, transition probabilities, lifetime, etc.) are related with the transitions between two levels, $\gamma^{\prime} J^{\prime}$ and $\gamma J$. The line strength between two levels can be written as

$$
S^{\pi k}\left(\gamma J, \gamma^{\prime} J^{\prime}\right)=\sum_{M, M^{\prime}, q}\left|\left\langle\gamma J M\left|O_{q}^{\pi(k)}\right| \gamma^{\prime} J^{\prime} M^{\prime}\right\rangle\right|^{2},
$$

where the transition operator $O_{q}^{\pi(k)}$ describes each multipole as a spherical tensor operator of rank $k$ and parity $\pi$. A transition probability that occurs between an upper state $\gamma^{\prime} J^{\prime}$ and lower state $\gamma J$ (emission transition) can be given by

$$
\begin{aligned}
& A^{\pi k}\left(\gamma^{\prime} J^{\prime}, \gamma J\right) \\
& \quad=2 C_{k}\left[\alpha\left(E_{\gamma ! J^{\prime}}-E_{\gamma J}\right)\right]^{2 k+1} \frac{S^{\pi k}\left(\gamma^{\prime} J^{\prime}, \gamma J\right)}{g_{\mathrm{J}^{\prime}}},
\end{aligned}
$$

where $g_{\mathrm{J}^{\prime}}$ is statistical weight of upper level $\left(g_{\mathrm{J}^{\prime}}=2 J^{\prime}+1\right)$ and $C_{k}$ is

$$
C_{k}=\frac{(2 k+1)(k+1)}{k((2 k+1) ! !)^{2}} \text {. }
$$

The oscillator strength may refer to transition either in absorption or emission. The absorption oscillator strength is

$$
\begin{aligned}
& f^{\pi k}\left(\gamma J, \gamma^{\prime} J^{\prime}\right) \\
& \quad=\frac{1}{\alpha} C_{k}\left[\alpha\left(E_{\gamma^{\prime} J^{\prime}}-E_{\gamma J}\right)\right]^{2 k-1} \frac{S^{\pi k}\left(\gamma J, \gamma^{\prime} J^{\prime}\right)}{g_{J}} .
\end{aligned}
$$

A similar expression can be written for the emission oscillator strength where $\gamma^{\prime} J^{\prime}$ and $\gamma J$ are interchanged, making the emission oscillator strength negative. The weighted oscillator strength, or $g f$-value, is completely symmetrical (except sign) between the two levels. The weighted oscillator strength is given by

$$
g f^{\pi k}\left(\gamma J, \gamma^{\prime} J^{\prime}\right)=g_{\mathrm{J}} f^{\pi k}\left(\gamma J, \gamma^{\prime} J^{\prime}\right) \text {. }
$$

The electric transitions have the form

$$
E_{q}^{(k)}=\sum_{i=1}^{N} r^{k}(i) C_{q}^{k}(i) .
$$

The largest transition is electric dipole $E^{(1)}$ radiation $\left(A \propto \alpha^{2 k+1}\right)$ where $k$ is 1 . If the parties of two levels are denoted by $\pi$ and $\pi^{\prime}$ and considered $\frac{\pi^{\prime}}{\pi}$, then

$$
E^{(k)}: \frac{\pi^{\prime}}{\pi}=(-1)^{k} .
$$

The electric dipole operator $E^{(1)}$ combines states of different parties.

\section{Results and discussion}

In this paper, we have reported the transition parameters including the excitation energies, wavelengths, oscillator strengths and transition probabilities of some low-lying levels for Ac I using the MCHF code within the framework Breit-Pauli Hamiltonian [21]. Correlation and the interelectronic relativistic interaction described by the Breit-Pauli Hamiltonian are important in precise calculations of heavy atoms, also including actinides. It is well known that Breit effects grow very rapidly with increasing nuclear charge. These make important contribution to the physical and chemical properties.

To consider correlation effects we have selected the configuration sets in Table I for understanding the correlation effect. We have to consider these effects for actinium due to complex spectra of actinides. In particular we tried to select the configurations including the $5 f$ and $5 g$ orbitals in the $B$ and $C$, respectively. The energies important for excitation may be calculated by increasing the number of configurations including filled $5 f$ shell. Because of the greater $5 f$ radii, $5 f-7 s$ and $5 f-7 p$ interactions tend to be stronger than the corresponding lanthanides [22]. But in this case, these configurations have complicated the calculations due to the computer constraints although we varied some parameter values increasingly in the MCHF code for considering the configurations mentioned in this work. We have not taken configuration sets including partially filled $5 \mathrm{f}$ subshell other than $5 f$ and $5 f^{2}$ due to the computer and the MCHF code constraints.

We have also calculated first ionization energy besides the excitation energies and electric dipole transition parameters. We have obtained the value $42698.86 \mathrm{~cm}^{-1}$ $(5.29 \mathrm{eV})$ for the first ionization energy of Ac I . In literature, the values of first ionization energy are given as $45730 \mathrm{~cm}^{-1}[9], 45849 \mathrm{~cm}^{-1}$ [10], $41700 \mathrm{~cm}^{-1}$ (5.17 eV [16], it can be also found in [2] and [23]), and $5.31 \mathrm{eV}$ [11]. Our result is in very good agreement especially with [9] and [11]. This agreement with these other works supports the reliability of our results. 
TABLE II

Energies [in $\mathrm{cm}^{-1}$ ] of $6 d 7 s^{2}, 6 d^{2} 7 s, 6 d 7 s 7 p$ and $6 d^{2} 7 p$ levels for Ac I .

\begin{tabular}{|c|c|c|c|c|c|c|}
\hline \multirow{2}{*}{\multicolumn{3}{|c|}{ Levels }} & \multicolumn{4}{|c|}{ Energies } \\
\hline & & & \multicolumn{3}{|c|}{ This work } & \multirow[t]{2}{*}{ Other work [1] } \\
\hline Conf. & Term & $J$ & $A$ & $B$ & $C$ & \\
\hline \multicolumn{7}{|c|}{ For even-parity: } \\
\hline \multirow[t]{2}{*}{$6 d 7 s^{2}$} & ${ }^{2} D$ & $3 / 2$ & 0.00 & 0.00 & 0.00 & 0.00 \\
\hline & & $5 / 2$ & 3271.59 & 2571.84 & 2830.00 & 2231.43 \\
\hline \multirow[t]{5}{*}{$6 d^{2}\left({ }^{3} F_{2}\right) 7 s$} & ${ }^{4} F$ & $3 / 2$ & 5623.92 & 6218.47 & 7086.83 & 9217.28 \\
\hline & & $5 / 2$ & 6569.00 & 7145.69 & 7987.41 & 9863.59 \\
\hline & & $7 / 2$ & 8239.19 & 8574.27 & 9517.43 & 10906.02 \\
\hline & & $9 / 2$ & 10250.86 & 10179.00 & 11129.04 & 12078.07 \\
\hline & ${ }^{2} F$ & $5 / 2$ & 10679.87 & 12339.86 & 12506.95 & - \\
\hline \multirow[t]{3}{*}{$6 d^{2}\left({ }^{3} P_{2}\right) 7 s$} & ${ }^{4} P$ & $1 / 2$ & 11640.25 & 12485.56 & 14229.97 & - \\
\hline & & $3 / 2$ & 11603.58 & 13485.34 & 15018.67 & - \\
\hline & & $5 / 2$ & 12433.90 & 13615.75 & 17583.20 & - \\
\hline $6 d^{2}\left({ }^{3} F_{2}\right) 7 s$ & ${ }^{2} F$ & $7 / 2$ & 14324.96 & 14714.01 & 14845.31 & - \\
\hline \multirow[t]{2}{*}{$6 d^{2}\left({ }^{3} P_{2}\right) 7 s$} & ${ }^{2} P$ & $1 / 2$ & 14727.33 & - & - & - \\
\hline & & $3 / 2$ & 16710.54 & - & & - \\
\hline $6 d^{2}\left({ }^{1} G_{2}\right) 7 s$ & ${ }^{2} G$ & $9 / 2$ & - & 15832.00 & 16461.13 & - \\
\hline \multirow[t]{2}{*}{$6 d^{2}\left({ }^{1} D_{2}\right) 7 s$} & ${ }^{2} D$ & $3 / 2$ & 12418.01 & 16000.93 & 17083.49 & - \\
\hline & & $5 / 2$ & - & 16386.03 & - & - \\
\hline $6 d^{2}\left({ }^{1} G_{2}\right) 7 s$ & ${ }^{2} G$ & $7 / 2$ & - & 16620.19 & - & - \\
\hline $6 d^{2}\left({ }^{1} S\right) 7 s$ & ${ }^{2} S$ & $1 / 2$ & - & 25581.35 & 28680.34 & - \\
\hline \multicolumn{7}{|c|}{ For odd-parity: } \\
\hline \multirow[t]{2}{*}{$6 d 7 s\left({ }^{3} D\right) 7 p$} & ${ }^{4} F$ & $3 / 2$ & 3715.46 & 15166.06 & 14055.77 & 13712.90 \\
\hline & & $5 / 2$ & 5082.17 & 15954.33 & 15015.83 & 14940.72 \\
\hline $6 d 7 s\left({ }^{3} D\right) 7 p$ & ${ }^{4} D$ & $1 / 2$ & 4388.23 & 17867.23 & 17456.62 & 17199.71 \\
\hline $6 d 7 s\left({ }^{1} D\right) 7 p$ & ${ }^{2} D$ & $3 / 2$ & - & 19166.36 & 18024.81 & 26066.04 \\
\hline \multirow[t]{3}{*}{$6 d 7 s\left({ }^{3} D\right) 7 p$} & ${ }^{4} F$ & $7 / 2$ & 5966.09 & 19187.19 & 17872.90 & 17683.87 \\
\hline & ${ }^{2} D$ & $3 / 2$ & 6719.56 & - & - & 17736.26 \\
\hline & ${ }^{4} D$ & $7 / 2$ & 7649.66 & 20720.98 & 20417.88 & 23475.94 \\
\hline \multirow[t]{3}{*}{$6 d^{2}\left({ }^{3} F_{2}\right) 7 p$} & ${ }^{4} G$ & $7 / 2$ & 10630.74 & 21018.70 & 22918.53 & 32219.62 \\
\hline & & & & & & 33756.43 \\
\hline & & & & & & 34360.25 \\
\hline \multirow[t]{3}{*}{$6 d 7 s\left({ }^{3} D\right) 7 p$} & ${ }^{4} P$ & $1 / 2$ & 6992.32 & 21516.94 & 20995.00 & 22401.52 \\
\hline & & $3 / 2$ & 7265.88 & 22119.14 & 21253.01 & 22801.10 \\
\hline & ${ }^{4} F$ & $9 / 2$ & 8110.04 & 22136.98 & 20790.62 & - \\
\hline $6 d^{2}\left({ }^{3} F_{2}\right) 7 p$ & ${ }^{4} F$ & $3 / 2$ & 9592.49 & 22918.83 & 23816.27 & - \\
\hline \multirow[t]{3}{*}{$6 d 7 s\left({ }^{3} D\right) 7 p$} & ${ }^{4} P$ & $5 / 2$ & 9785.15 & 23233.45 & 22359.34 & 23898.86 \\
\hline & ${ }^{2} F$ & $5 / 2$ & 17214.43 & - & 23327.48 & 23916.84 \\
\hline & & $7 / 2$ & 13336.80 & - & 24266.71 & 24969.30 \\
\hline \multirow[t]{2}{*}{$6 d 7 s\left({ }^{1} D\right) 7 p$} & ${ }^{2} F$ & $5 / 2$ & - & - & 36309.08 & 26836.20 \\
\hline & & $7 / 2$ & - & - & 37915.44 & 28568.40 \\
\hline \multirow[t]{4}{*}{$6 d^{2}\left({ }^{3} F_{2}\right) 7 p$} & ${ }^{4} G$ & $9 / 2$ & 11245.86 & 23646.32 & 23282.43 & 32867.39 \\
\hline & & & & & & 34788.12 \\
\hline & & & & & & 35870.00 \\
\hline & ${ }^{4} F$ & $7 / 2$ & 11643.26 & 25082.44 & 25257.28 & - \\
\hline $6 d 7 s\left({ }^{1} D\right) 7 p$ & ${ }^{2} P$ & $3 / 2$ & - & 25479.60 & - & - \\
\hline $6 d^{2}\left({ }^{3} F_{2}\right) 7 p$ & ${ }^{4} F$ & $9 / 2$ & 13721.45 & 25896.89 & 26143.18 & - \\
\hline & ${ }^{4} G$ & $11 / 2$ & 13352.31 & 26109.94 & 25682.95 & 33429.76 \\
\hline & ${ }^{2} F$ & $5 / 2$ & - & 27144.98 & 27662.59 & - \\
\hline & ${ }^{4} D$ & $3 / 2$ & 13037.98 & 27455.76 & 28254.50 & - \\
\hline $6 d^{2}\left({ }^{3} P_{2}\right) 7 p$ & ${ }^{4} D$ & $1 / 2$ & 16654.95 & 28088.48 & 27938.21 & - \\
\hline $6 d^{2}\left({ }^{3} F_{2}\right) 7 p$ & ${ }^{4} D$ & $5 / 2$ & 14018.33 & 28522.30 & 29123.49 & - \\
\hline & ${ }^{2} G$ & $9 / 2$ & 16721.27 & - & - & - \\
\hline $6 d^{2}\left({ }^{3} P_{2}\right) 7 p$ & ${ }^{4} D$ & $3 / 2$ & 19402.02 & 28879.80 & 28931.90 & - \\
\hline
\end{tabular}


TABLE II (cont.)

\begin{tabular}{|c|c|c|c|c|c|c|}
\hline \multirow{2}{*}{\multicolumn{3}{|c|}{ Levels }} & \multicolumn{4}{|c|}{ Energies } \\
\hline & & & \multicolumn{3}{|c|}{ This work } & \multirow[t]{2}{*}{ Other work [1] } \\
\hline Conf. & Term & $J$ & $A$ & $B$ & $C$ & \\
\hline & ${ }^{2} S$ & $1 / 2$ & 15348.50 & 29172.36 & 28897.75 & - \\
\hline & ${ }^{2} D$ & $3 / 2$ & 26459.02 & 29655.01 & 31804.37 & - \\
\hline $6 d 7 s\left({ }^{3} D\right) 7 p$ & ${ }^{2} P$ & $1 / 2$ & - & 30825.50 & 30390.51 & 25729.03 \\
\hline \multirow[t]{4}{*}{$6 d^{2}\left({ }^{3} P_{2}\right) 7 p$} & ${ }^{4} D$ & $7 / 2$ & 19698.54 & 32014.36 & 32101.57 & - \\
\hline & ${ }^{2} D$ & $5 / 2$ & 18789.02 & 46101.40 & - & - \\
\hline & ${ }^{4} P$ & $1 / 2$ & 19125.59 & 32344.64 & 32227.43 & - \\
\hline & & $3 / 2$ & - & 32814.30 & 31172.48 & - \\
\hline $6 d^{2}\left({ }^{1} G_{2}\right) 7 p$ & ${ }^{2} G$ & $7 / 2$ & 20781.26 & - & - & - \\
\hline \multirow[t]{2}{*}{$6 d^{2}\left({ }^{1} D_{2}\right) 7 p$} & ${ }^{2} D$ & $3 / 2$ & 22206.70 & - & - & - \\
\hline & & $5 / 2$ & 23513.47 & - & - & - \\
\hline \multirow[t]{2}{*}{$6 d^{2}\left({ }^{1} G_{2}\right) 7 p$} & ${ }^{2} H$ & $11 / 2$ & 22563.39 & 33370.01 & 32272.32 & - \\
\hline & & $9 / 2$ & 21954.28 & 33903.96 & - & - \\
\hline \multirow[t]{2}{*}{$6 d^{2}\left({ }^{3} P_{2}\right) 7 p$} & ${ }^{2} P$ & $3 / 2$ & 31501.19 & 34931.06 & 35066.33 & - \\
\hline & & $1 / 2$ & 29429.59 & 35548.52 & 35877.25 & - \\
\hline \multirow[t]{2}{*}{$6 d^{2}\left({ }^{1} D_{2}\right) 7 p$} & ${ }^{2} F$ & $5 / 2$ & 22659.79 & 36496.98 & - & - \\
\hline & & $7 / 2$ & 23293.54 & 36657.91 & - & - \\
\hline $6 d^{2}\left({ }^{1} G_{2}\right) 7 p$ & ${ }^{2} G$ & $9 / 2$ & 20985.65 & 36946.24 & - & - \\
\hline $6 d 7 s\left({ }^{3} D\right) 7 p$ & ${ }^{4} D$ & $3 / 2$ & 4655.98 & 19698.97 & 16283.76 & 19012.46 \\
\hline $6 d^{2}\left({ }^{1} D_{2}\right) 7 p$ & ${ }^{2} P$ & $1 / 2$ & 22862.77 & 37530.68 & 36925.60 & - \\
\hline $6 d 7 s\left({ }^{3} D\right) 7 p$ & ${ }^{4} D$ & $5 / 2$ & 7427.63 & 19562.84 & 18834.66 & 21195.87 \\
\hline \multirow[t]{4}{*}{$6 d^{2}\left({ }^{3} F_{2}\right) 7 p$} & ${ }^{4} G$ & $5 / 2$ & 9222.70 & 21621.73 & 21153.56 & 31494.68 \\
\hline & & & & & & 32495.67 \\
\hline & & & & & & 34658.47 \\
\hline & ${ }^{4} F$ & $5 / 2$ & 10471.64 & 24172.17 & 24408.46 & - \\
\hline \multirow[t]{2}{*}{$6 d^{2}\left({ }^{1} S_{0}\right) 7 p$} & ${ }^{2} P$ & $1 / 2$ & 36983.06 & - & 46000.22 & - \\
\hline & & $3 / 2$ & 37972.91 & 49732.39 & 49518.17 & - \\
\hline \multirow[t]{7}{*}{$6 d^{2}\left({ }^{3} F_{2}\right) 7 p$} & ${ }^{2} G$ & $7 / 2$ & 13997.27 & 25516.06 & 26445.64 & - \\
\hline & ${ }^{4} D$ & $1 / 2$ & 12691.17 & 27333.78 & 28209.45 & - \\
\hline & & $7 / 2$ & - & 28358.58 & - & - \\
\hline & ${ }^{2} D$ & $3 / 2$ & 8238.59 & 33850.68 & 36186.00 & - \\
\hline & ${ }^{4} D$ & $7 / 2$ & 15333.94 & 29819.04 & 30118.61 & - \\
\hline & & $5 / 2$ & 18136.01 & 30551.49 & 30056.72 & - \\
\hline & ${ }^{2} D$ & $5 / 2$ & 11017.50 & 35846.16 & 37893.21 & - \\
\hline $6 d^{2}\left({ }^{3} P_{2}\right) 7 p$ & ${ }^{4} S$ & $3 / 2$ & 17847.17 & 32045.19 & 30831.20 & - \\
\hline $6 d^{2}\left({ }^{1} D_{2}\right) 7 p$ & ${ }^{2} P$ & $3 / 2$ & 21443.11 & 25479.60 & 25101.80 & - \\
\hline $6 d^{2}\left({ }^{3} P_{2}\right) 7 p$ & ${ }^{4} P$ & $5 / 2$ & 19966.50 & 31804.25 & 31961.99 & - \\
\hline \multirow[t]{2}{*}{$6 d^{2}\left({ }^{1} G_{2}\right) 7 p$} & ${ }^{2} F$ & $7 / 2$ & 29081.98 & 37690.79 & 39634.64 & - \\
\hline & & $5 / 2$ & 27072.22 & 39289.99 & 39667.25 & - \\
\hline
\end{tabular}

The relativistic energies $(\mathrm{MCHF}+\mathrm{BP})$ for $6 d 7 s^{2}$ and $6 d^{2} 7 s$ even-parity levels and $6 d 7 s 7 p$ and $6 d^{2} 7 p$ odd-parity levels have been reported in Table II. The level energies are relative to the ground state $6 d 7 s^{2}{ }^{2} D_{3 / 2}$. It is seen that the agreement is not very poor when our results are compared with the other works. In particular, the calculation results obtained according to $B$ and $C$ configuration sets are generally good. The same results for $6 d^{2} 7 p$ are somewhat poor. $7 s^{2} 7 p^{2} P_{1 / 2}$ level according to $C$ configuration set is lower than $6 d 7 s 7 p^{4} F_{3 / 2}$ level which is lower odd-parity level in literature. The excitation energy of $7 s^{2} 7 p^{2} P_{1 / 2}$ is $12686.36 \mathrm{~cm}^{-1}$. Thus it is seen that the results are better when $5 f$ orbital is included in configuration set.

In Table III, there are given the electric dipole $E^{(1)}$ transition data including wavelengths, $\lambda(\AA)$, weighted oscillator strengths, $g f$-values, and transition probabilities (or rates), $A_{k i}\left(\mathrm{~s}^{-1}\right)$, for $6 d 7 s^{2}-6 d 7 s 7 p$ transition. The results presented in this table have been obtained by $B$ and $C$ configuration sets. The level energies obtained using these sets are better than in corresponding $A$ configuration set. When a comparison of wavelengths for this transition has been made with existing values in literature, our results are in agreement. 
TABLE III

Wavelengths, $\lambda[\AA]$, weighted oscillator strengths, $g f$-values, and transition probabilities, $A_{k i}\left[\mathrm{~s}^{-1}\right]$, for $6 d 7 s^{2}-6 d 7 s 7 p$ transition in Ac I . The superscripts " $a$ " and " $b$ " represent Ref. [1] and Ref. [2], respectively. The wavelength values taken from Ref. [1] are here converted to $\AA$ unit.

\begin{tabular}{|c|c|c|c|c|}
\hline \multirow{2}{*}{ Transitions } & \multicolumn{2}{|c|}{$\lambda$} & \multirow{2}{*}{$g f$-values } & \multirow{2}{*}{$A_{k i}$} \\
\hline & This work & Other works & & \\
\hline $6 d 7 s^{2}{ }^{2} D_{3 / 2}-6 d 7 s\left({ }^{3} D\right) 7 p^{2} P_{1 / 2}$ & $\begin{array}{l}3264.85^{B} \\
3307.04^{C}\end{array}$ & $3885.56^{b}$ & $\begin{array}{l}6.38 \times 10^{-1} B \\
5.56 \times 10^{-1} C\end{array}$ & $\begin{array}{l}1.99 \times 10^{8 B} \\
1.69 \times 10^{8} \mathrm{C}\end{array}$ \\
\hline $6 d 7 s^{2}{ }^{2} D_{3 / 2}-6 d 7 s\left({ }^{3} D\right) 7 p^{2} F_{5 / 2}$ & $4314.90^{C}$ & $4179.98^{a, b}$ & $2.59 \times 10^{-1 C}$ & $1.55 \times 10^{7} \mathrm{C}$ \\
\hline $6 d 7 s^{2}{ }^{2} D_{3 / 2}-6 d 7 s\left({ }^{3} D\right) 7 p{ }^{4} P_{5 / 2}$ & $\begin{array}{l}4340.81^{B} \\
4503.01^{C}\end{array}$ & $4183.12^{a, b}$ & $\begin{array}{l}8.89 \times 10^{-2} B \\
9.17 \times 10^{-2} C\end{array}$ & $\begin{array}{l}5.24 \times 10^{6} B \\
5.03 \times 10^{6} C\end{array}$ \\
\hline $6 d 7 s^{2}{ }^{2} D_{5 / 2}-6 d 7 s\left({ }^{1} D\right) 7 p^{2} D_{3 / 2}$ & $\begin{array}{l}6098.21^{B} \\
6647.69^{C}\end{array}$ & $4194.40^{a, b}$ & $\begin{array}{l}7.06 \times 10^{-2} B \\
7.50 \times 10^{-3} C\end{array}$ & $\begin{array}{l}3.16 \times 10^{6} B \\
2.83 \times 10^{5} \mathrm{C}\end{array}$ \\
\hline $6 d 7 s^{2}{ }^{2} D_{3 / 2}-6 d 7 s\left({ }^{3} D\right) 7 p{ }^{4} P_{3 / 2}$ & $\begin{array}{l}4561.44^{B} \\
4739.11^{C}\end{array}$ & $4384.53^{a, b}$ & $\begin{array}{l}2.80 \times 10^{-2} B \\
9.72 \times 10^{-3} C\end{array}$ & $\begin{array}{l}2.24 \times 10^{6} B \\
7.22 \times 10^{5} C\end{array}$ \\
\hline $6 d 7 s^{2}{ }^{2} D_{5 / 2}-6 d 7 s\left({ }^{3} D\right) 7 p^{2} F_{7 / 2}$ & $4698.20^{C}$ & $4396.71^{a, b}$ & $2.12 \times 10^{-1 C}$ & $8.02 \times 10^{6 C}$ \\
\hline $6 d 7 s^{2}{ }^{2} D_{3 / 2}-6 d 7 s\left({ }^{3} D\right) 7 p{ }^{4} P_{1 / 2}$ & $\begin{array}{c}10841.79^{B} \\
7269.45^{C}\end{array}$ & $4462.73^{a, b}$ & $\begin{array}{l}3.67 \times 10^{-3} B \\
1.86 \times 10^{-3} C\end{array}$ & $\begin{array}{l}1.04 \times 10^{5} B \\
1.17 \times 10^{5 C}\end{array}$ \\
\hline $6 d 7 s^{2}{ }^{2} D_{5 / 2}-6 d 7 s\left({ }^{3} D\right) 7 p{ }^{4} P_{5 / 2}$ & $5160.66^{C}$ & $4613.93^{b}$ & $9.64 \times 10^{-2 C}$ & $4.02 \times 10^{6 C}$ \\
\hline $6 d 7 s^{2}{ }^{2} D_{3 / 2}-6 d 7 s\left({ }^{3} D\right) 7 p{ }^{4} D_{5 / 2}$ & $\begin{array}{l}5163.53^{B} \\
5352.55^{C}\end{array}$ & $4716.58^{a, b}$ & $\begin{array}{l}1.08 \times 10^{-3} B \\
8.73 \times 10^{-3} C\end{array}$ & $\begin{array}{l}4.50 \times 10^{4} B \\
3.38 \times 10^{5} C\end{array}$ \\
\hline $6 d 7 s^{2}{ }^{2} D_{3 / 2}-6 d 7 s\left({ }^{3} D\right) 7 p{ }^{4} D_{3 / 2}$ & $\begin{array}{l}5127.49^{B} \\
6198.94^{C}\end{array}$ & $5258.24^{b}$ & $\begin{array}{l}1.73 \times 10^{-1} B \\
2.59 \times 10^{-3} C\end{array}$ & $\begin{array}{l}1.10 \times 10^{7} B \\
1.12 \times 10^{5} \mathrm{C}\end{array}$ \\
\hline $6 d 7 s^{2}{ }^{2} D_{3 / 2}-6 d 7 s\left({ }^{3} D\right) 7 p{ }^{4} F_{5 / 2}$ & $\begin{array}{l}6345.95^{B} \\
6727.73^{C}\end{array}$ & $6691.27^{a, b}$ & $\begin{array}{l}1.84 \times 10^{-1} B \\
1.19 \times 10^{-1} C\end{array}$ & $\begin{array}{l}5.09 \times 10^{6} B \\
2.93 \times 10^{6} \mathrm{C}\end{array}$ \\
\hline $6 d 7 s^{2}{ }^{2} D_{3 / 2}-6 d 7 s\left({ }^{3} D\right) 7 p{ }^{4} F_{3 / 2}$ & $\begin{array}{l}6680.11^{B} \\
7192.28^{C}\end{array}$ & $7290.40^{a, b}$ & $\begin{array}{l}8.27 \times 10^{-2} B \\
5.84 \times 10^{-2} C\end{array}$ & $\begin{array}{l}3.09 \times 10^{6} B \\
1.88 \times 10^{6} \mathrm{C}\end{array}$ \\
\hline
\end{tabular}

In this work, we have considered only valence correlation where two valence orbitals are excited. If corevalence $(\mathrm{CV})$ correlation where one core orbital and one valence orbital and core-core (CC) correlation where two core orbitals are excited, are taken, the transition results will be better because the transition calculations depend on total energy differences. But, CV and CC correlation models produce a lot of CSFs. In this case, the computer capacity problems occur for Ac I . In addition, we have made an application of $\mathrm{MCHF}+\mathrm{BP}$ method for actinium. We have obtained good results for excited levels in our previous works for some lanthanide atoms (La and recently Lu) using this approach. Of course, for elements with intermediate atomic number, the perturbation theory such as the Breit-Pauli Hamiltonian which allows one to examine the various relativistic terms on the basis of the Schrödinger wave function and to include these corrections. In the $\mathrm{MCHF}+\mathrm{BP}$ approach, the relativistic effects are taken as the difference between the Dirac and Schrödinger equation for a given system. In this work, we have taken this approach for considering the excitation of three electrons in valence shell although actinium is a heavier element. In such systems it is sufficient, to the route, to consider only the lowest-order relativistic corrections to the ordinary Schrödinger equation (Cowan [22] and Fischer [17]). These corrections can be derived from the relativistic many-electron equations by expanding in powers of $1 / c$, where $c$ is the speed of light.

We hope that these data for Ac I are very useful because the information about the spectrum of this atom and actinide series is very fragmentary in literature. We reported new data including valence correlation and relativistic corrections in Ac I . It is known that the experiments are extremely expensive and difficult and the theoretical methods need huge computing facilities or long time to be worked out for heavy elements such as actinium. There are a few experimental or theoretical wavelengths, oscillator strengths $(g f)$ and transition probabilities $\left(A_{k i}\right)$ values for this atom in literature. Therefore, one of the purposes of performing these calculations is to apply them in spectrum which provides useful information. Reliable atomic data in the study of astrophysical problems are needed. Recently, the experimental investigation for physical and chemical properties of heavy elements including actinides is being developed due to the synthesis of elements from the "island of stability". Therefore we think that these computational data will be various physical and chemical fields such as the studies of nucleusynthesis of heavy elements and the investigation of spectra for rare-earth elements.

\section{Acknowledgments}

The authors are very grateful to the anonymous reviewers for stimulating comments and valuable suggestions, which resulted in improving the presentation of the paper. 


\section{References}

[1] http://www.lac.u-psud.fr/Database/Contents.html .

[2] http://physics.nist.gov/PhysRefData/ ASD/index.html.

[3] B.R. Judd, Phys. Rev. 125, 613 (1962).

[4] L.J. Nugent, K.L. Vander Sluis, J. Opt. Soc. Am. 61, 1112 (1971).

[5] K.L. Vander Sluis, L.J. Nugent, Phys. Rev. A 6, 86 (1972).

[6] L. Brewer, J. Opt. Soc. Am. 61, 1666 (1971).

[7] J.P. Desclaux, At. Data Nucl. Data Tables 12, 311 (1973).

[8] J.B. Mann, At. Data Nucl. Data Tables 12, 1 (1973).

[9] K. Rajnak, J. Opt. Soc. Am. 68, 360 (1978).

[10] S. Köhler, R.D. Benberger, K. Eberhart, N. Erdmann, G. Herrmann, G. Huber, J.V. Kratz, M. Nunnemann, G. Passler, P.M. Rao, J. Riegel, N. Trautmann, K. Wendt, Spectrochim. Acta B 52, 717 (1997).

[11] E. Eliav, S. Shmulyian, U. Kaldor, Y. Ishikawa, J. Chem. Phys. 109, 3954 (1998).

[12] M. Seth, K.G. Dyall, R. Sherpord, A. Wagner, J. Phys. B, At. Mol. Opt. Phys. 34, 2383 (2001).
[13] A.N. Petrov, N.S. Mosyagin, A.V. Titov, I.I. Tupitsyn, J. Phys. B, At. Mol. Opt. Phys. 37, 4621 (2004).

[14] P.S. Doidge, Spectrochim. Acta B 50, 209 (1995).

[15] J. Sugar, J. Chem. Phys. 59, 788 (1973).

[16] J. Sugar, J. Chem. Phys. 60, 4103 (1974).

[17] C.F. Fischer, T. Brage, P. Jönsson, Computational Atomic Structure - an MCHF Approach, Institute of Physics Publ., Bristol 1997.

[18] B. Karaçoban, L. Özdemir, J. Quant. Spectrosc. Radiat. Transfer 109, 1968 (2008).

[19] B. Karaçoban, L. Özdemir, Acta Phys. Pol. A 113, 1609 (2008).

[20] B. Karaçoban, L. Özdemir, Acta Phys. Pol. A 115, 864 (2009).

[21] C.F. Fischer, Comput. Phys. Commun. 64, 369 (1991).

[22] R.C. Cowan, The Theory of Atomic Structure and Spectra, University of California Press, London 1981.

[23] Handbook of Chemistry and Physics, 78th ed., Ed. D.R. Lide, CRC, Boca Raton, Florida 1997. 